\title{
Design and calculation of photoelectric properties of type-II superlattice photodetectors with diffraction ring structure
}

\section{Yanan Du}

Institute of Semiconductors, Chinese Academy of Sciences

Yun Xu ( $\nabla$ xuyun@semi.ac.cn )

Institute of Semiconductors, Chinese Academy of Sciences

Bo Cheng

Institute of Semiconductors, Chinese Academy of Sciences

Guofeng Song

Institute of Semiconductors, Chinese Academy of Sciences

\section{Research Article}

Keywords: type-Il superlattices, diffraction ring, resonant cavity, quantum efficiency

Posted Date: April 20th, 2021

DOI: https://doi.org/10.21203/rs.3.rs-424259/v1

License: (c) (i) This work is licensed under a Creative Commons Attribution 4.0 International License.

Read Full License 


\title{
Design and calculation of photoelectric properties of type-II superlattice photodetectors with diffraction ring structure
}

\author{
Yanan $\mathrm{Du}^{1,2,3}$, Yun $\mathrm{Xu}{ }^{1,2,3, *}$, Bo Cheng ${ }^{1,2,3}$, Guofeng Song ${ }^{1,2,3}$ \\ 1 Institute of Semiconductors, Chinese Academy of Sciences, Beijing, 100083, China \\ 2 College of Materials Science and Opto-Electronic Technology, University of Chinese Academy of Sciences, \\ Beijing, 100049, China \\ 3 Beijing Key Laboratory of Inorganic Stretchable and Flexible Information Technology, Beijing 100083, China.
}

*Corresponding author. E-mail: xuyun@semi.ac.cn

\begin{abstract}
The photoelectric properties of type-II superlattices (T2SLs) infrared detectors with diffraction ring resonance structure are calculated and studied in this paper. In order to improve the quantum efficiency (QE), diffraction rings are designed on the top surface of T2SLs detectors to control the incident light inside. The maximum QE of ordinary detector is about $30 \%$, while the detector with square diffraction rings is about $80 \%$, and the detector with circular diffraction rings is about $60 \%$. It shows that the structures with diffraction ring can significantly improve the QE of photodetectors. In addition, the size and shape of the resonator are studied. it is obvious that the optimization effect of the square diffraction ring is better than that of the circular diffraction ring, and the square diffraction ring is insensitive to the size change, so it is suitable for the manufacture of practical photodetectors. At the same time, the dark current mechanism of the detector is analyzed and the corresponding specific detectivity is calculated.
\end{abstract}

Keyword: type-II superlattices; diffraction ring; resonant cavity; quantum efficiency

\section{Introduction}

T2SLs have been widely used in photodetectors since they were proposed in $1977^{1}$. T2SLs have tunable energy band, and the corresponding cut-off wavelength is in the wavelength range of $3 \sim 32 \mu \mathrm{m}^{2}$. T2SLs have special band structures. Taking InAs/GaSb T2SLs as an example, due to the special band alignment, electrons and holes are confined in InAs and GaSb layers respectively by ultra-thin layers of superlattices, and then minibands formed ${ }^{3}$. The electrons absorb the incident light photons and transits between the minibands, resulting in the generation of photogenerated carriers, which are collected by the electrodes to form photocurrent signals under the built-in electric field. The main optimization directions of photodetectors include reducing dark current and increasing QE. In the device design, when the material is determined and the absorption coefficient is the intrinsic parameter of the material, increasing the thickness of the absorption layer within the diffusion length can increase the QE, but increase the dark current at the same time. So we consider the cavity structure, which can improve the QE without increasing the dark current.

The diffraction ring structure was designed and applied in QWIP by J. Sun and K. K. Choi et al ${ }^{4}$. It significantly improves the photoelectric performance of QWIP devices, but it is rarely used in T2SLs devices. The diffraction ring structure of resonant element can control the incident light inside the device and improve the QE, which is one of the good choices to optimize the performance of the devices. 
In this paper, the band theory model, device parameters and electromagnetic field model are established, and then the QE of 14.5ML InAs/7ML GaSb T2SLs PIN structure devices with and without resonators are calculated. Furthermore, the influence of the shape and size of the diffraction ring and the thickness of the absorption layer on the devices is calculated. The results show that the square diffraction ring structure has better optimization effect. The dark current mechanism of PIN structure is also analyzed. The theoretical model is established in Section 2. Results and discussion in Section 3. Summarize in Section 4.

\section{Theoretical methods}

\subsection{8-band $k \cdot p$ method}

In the design of T2SLs infrared detectors, the calculation of the band structure of the absorption layer is one of the important links. The calculated bands include the first conduction band, the first heavy hole band, the first light hole band and the band gap $\left(\mathrm{E}_{\mathrm{g}}\right)$. In this paper, the 8-band $\mathbf{k} \cdot \mathbf{p}$ method is used and the electron spin-orbit interaction is considered. The energy operator of electron spin-orbit interaction is added to the single electron Hamiltonian operator. The Schrodinger equation of Bloch function is ${ }^{5}$

$$
\left\{\frac{\hat{p}^{2}}{2 m_{0}}+V(r)+\frac{\hbar}{m_{0}} \boldsymbol{k} \cdot \widehat{\boldsymbol{p}}+\frac{\hbar}{4 m_{0}^{2} c_{0}^{2}}(\widehat{\boldsymbol{\sigma}} \times \nabla V) \cdot(\hat{\boldsymbol{p}}+\hbar \boldsymbol{k})\right\} \psi_{n \boldsymbol{k}}(r)=\left[E(\boldsymbol{k})-\frac{\hbar^{2} \boldsymbol{k}^{2}}{2 m_{0}}\right] \psi_{n \boldsymbol{k}}(r) .
$$

The Luttinger Kohn basis vector corresponding to $8 \times 8$ Hamiltonian matrix is derived from the transformation of Bloch wave function basis vector. The wave function can be written as a linear combination of envelope functions and basis vectors, which is as follows ${ }^{6}$

$$
\psi_{n k}(r)=\sum_{i=1}^{8} e^{i k_{t} r} u_{n}(r) F_{n}\left(k_{t}, z\right) .
$$

At the determined $k_{t}$, the eigen states and eigen bands can be solved by the following formula

$$
H\left(k_{t}\right) F_{n}\left(k_{t}, z\right)=E\left(k_{t}\right) F_{n}\left(k_{t}, z\right) .
$$

Periodic boundary conditions and finite difference method are used in the calculation. When $k_{t}$ is zero, the energy band at $\Gamma$ point in reciprocal space is calculated, which corresponds to the equivalent direct band gap position of T2SLs material, and the band gap width is obtained. All calculated temperatures in this paper are taken as $77 \mathrm{~K}$.

\subsection{Dark current and specific detectivity}

The dark current of photovoltaic PIN structure infrared detector mainly consists of thermal diffusion current $I_{\text {diff }}$, generate-recombination current $I_{g r}$, band to band tunneling current $I_{b t b}$, trap assisted tunneling current $I_{t a t}$ and leakage current $I_{\text {Rshunt }}$.

The thermal diffusion dark current generated by thermal excitation is one of the important dark current mechanisms in PIN junction devices. The formula of diffusion dark current is as follows ${ }^{7-9}$

$$
J_{\text {diff }}=q\left(\frac{L_{e} n_{i}^{2}}{\tau_{e} N_{A}}+\frac{L_{h} n_{i}^{2}}{\tau_{h} N_{D}}\right)\left[\exp \left(\frac{q V}{k_{B} T}\right)-1\right],
$$

where, $q$ is the electron charge and $k_{B}$ is the Boltzmann constant, $T$ is the Kelvin temperature, $V$ is the applied bias voltage; $\tau_{e}$ and $\tau_{h}$ are the minority carrier lifetimes of electrons and holes, and the values are $100 \mathrm{~ns}{ }^{10} ; L_{e}$ and $L_{h}$ are the diffusion lengths of electrons and holes, and $L_{e, h}=\sqrt{\frac{k T}{q} \mu_{e, h} \tau_{e, h}}$, where $\mu_{e}\left(\mu_{h}\right)$ is mobility of electrons in the p-region (holes in the n-region), and the values are 3000 and $300 \mathrm{~cm}^{2} /(\mathrm{V} \cdot \mathrm{s})$, respectively ${ }^{11} ; N_{A}$ and $N_{D}$ are the concentrations of acceptors and donors, and the specific values are given in the third part of device design, $n_{i}$ is the intrinsic carrier concentration.

The generation combination dark current is caused by the recombination process of carrier generation caused by depletion defects, and it is also a main dark current mechanism in PIN devices. The expression is as follows ${ }^{7-9}$

$$
J_{g r}=\frac{q n_{i} W}{\tau_{g r}} \frac{2 k_{B} T}{q\left(V_{b i}-V\right)} \sinh \left(\frac{q V}{2 k_{B} T}\right) f(b),
$$


where, $\tau_{g r}$ is the generation combined minority carrier lifetime, $V_{b i}$ is the built-in electric field, $W$ is the width of the depletion region. The specific formula of $\mathrm{F}(\mathrm{b})$ is as follows

$$
\begin{gathered}
f(b)=\left\{\begin{array}{cc}
\frac{1}{2 \sqrt{b^{2}-1}} \ln \left(2 b^{2}+2 b \sqrt{b^{2}-1}-1\right), b>1 \\
1, \quad b=1 \\
\frac{1}{\sqrt{1-b^{2}}} \tan ^{-1}\left(\frac{\sqrt{1-b^{2}}}{b}\right), & b<1
\end{array}\right. \\
b=\exp \left(-\frac{q V}{2 k_{B} T}\right) \cosh \left(\frac{E_{t}-E_{i}}{k_{B} T}\right),
\end{gathered}
$$

where, $E_{t}$ is trap state energy, $E_{i}$ is Fermi level.

For the band to band tunneling current, the expression is 7,9

$$
J_{b t b}=\frac{q^{3} \sqrt{2 m_{e}^{*} E V}}{4 \pi^{2} \hbar^{2} \sqrt{E_{g}}} \exp \left(-\frac{4 \sqrt{2 m_{e}^{*} E_{g}^{3}}}{3 q E \hbar}\right),
$$

where, $m_{e}^{*}$ is effective mass of electron, $E$ is electric field in depletion region, $\hbar$ is reduced Planck constant, $E_{g}$ is band gap of absorption layer, which is the difference between the first conduction band and the first hole band at $k_{t}=0$ calculated by $\mathbf{k} \cdot \mathbf{p}$ perturbation theory.

For the trap assisted tunneling dark current, the expression is as follows is $7,9,12$

$$
J_{\text {tat }}=\frac{q^{2} m_{e}^{*} V M^{2} N_{t}}{8 \pi \hbar^{3}} \exp \left(-\frac{4 \sqrt{2 m_{e}^{*}\left(E_{g}-E_{t}\right)^{3}}}{3 q E \hbar}\right),
$$

where, $M^{2}$ is matrix elements of trap potential with the value of $10^{-23} \mathrm{eV}^{2} \mathrm{~cm}^{39}, N_{t}$ is trap density.

The expression of specific detectivity is ${ }^{13}$

$$
D^{*}=\frac{\lambda \eta e}{2 \pi \hbar c} \sqrt{\frac{R_{0} A}{4 k_{B} T}} .
$$

where, $\eta$ is quantum efficiency, $c$ is light speed. $R_{0} A$ is the area product of differential resistance under zero bias voltage, which can be derived from dark current formula.

\subsection{Device design}

The modeling method in this paper uses the finite element method (FEM) to solve Maxwell's equation, get the electric field distribution, and then further get the QE. The Maxwell equation used is as follows

$$
\nabla \times\left(\mu_{r}^{-1} \nabla \times \boldsymbol{E}\right)-k_{0}^{2} \varepsilon_{r c} \boldsymbol{E}=0,
$$

where, $\mu_{r}$ is the relative permeability; $\varepsilon_{r c}$ is the relative dielectric constant, $\varepsilon_{r c}=\varepsilon_{r}-j \varepsilon_{i} ; k_{0}=2 \pi / \lambda$ is the free space wave vector. Periodic boundary conditions are used here.

The quantum efficiency of the detector is simulated as follows ${ }^{14}$

$$
\eta(\lambda)=\frac{n(\lambda) \alpha(\lambda)}{A E_{0}^{2}} \int_{V_{a c t}}|E(\boldsymbol{r}, \lambda)|^{2} d^{3} r,
$$

where, $A$ is the device area, $E_{0}$ is the incident electric field, $E$ is the electric field. Because the type II superlattice has no angle requirement for the incident light, the total electric field $E^{2}=E_{x}^{2}+E_{y}^{2}+E_{z}^{2}$, where $n(\lambda)$ is the refractive index of the material, $\alpha(\lambda)$ is the absorption coefficient, $V_{a c t}$ is the active volume of the device.

\section{Results and discussion}

The device design is shown in Fig. 1 (a). The device consists of a $500 \mathrm{~nm}$ GaSb buffer layer, a $650 \mathrm{~nm}$ (100 periods) 14.5ML InAs/ 7ML GaSb T2SLs p region (p-type doping concentration $10^{18} \mathrm{~cm}^{-3}$ ), a $1950 \mathrm{~nm}$ (300 periods) unintentionally doped 14.5ML InAs/ 7ML GaSb T2SLs absorption layer $\left(5 \times 10^{16} \mathrm{~cm}^{-3}\right)$, a $650 \mathrm{~nm}$ (100 periods) 14.5ML InAs/ 7ML GaSb T2SLs n region (n-type doping concentration $\left.10^{18} \mathrm{~cm}^{-3}\right)$. The metal layer is Ti $(100 \mathrm{~nm})$ 
$/ \mathrm{Au}(300 \mathrm{~nm})$ metal layer. The mesa size is $30 \times 30 \mu \mathrm{m}$, and the top and bottom metal layers are $26 \times 26 \mu \mathrm{m}$ and $15 \times 3$ $\mu \mathrm{m}$, respectively. The diffraction ring is made of GaSb bulk material. The outer diameter of the diffraction ring is 4.5 $\mu \mathrm{m}$, the inner diameter is $3 \mu \mathrm{m}$, the height is $0.35 \mu \mathrm{m}$, and the spacing of each diffraction ring is $8 \mu \mathrm{m}$. The first conduction band (C1) and first heavy hole band (HH1) of 14.5ML InAs/ 7ML GaSb T2SLs are calculated by k·p perturbation theory. The wave functions are shown in Fig. 1 (b), and the $50 \%$ cutoff wavelength is about $9 \mu \mathrm{m}$. The absorption coefficient data are from reference ${ }^{6}$. First of all, the electromagnetic field of the device without diffraction ring is simulated by COMSOL software, and QE is calculated, which is compared with the calculation results of the formula ${ }^{15}$ and the quantum efficiency of the material with similar structure and size ${ }^{6}$, as shown in Fig. 1 (c). In COMSOL software simulation, the interlayer oscillation, light reflection and transmission are considered, so the calculation results have oscillation peaks and the value is less than the theoretical formula value, so it can be considered that the simulation results are relatively correct. The electromagnetic field model is used to simulate the electromagnetic field of the device with square diffraction ring, circular diffraction ring, diamond diffraction ring and no diffraction ring, and the comparison results are shown in Fig. 1 (d). Obviously, the square diffraction ring has a better effect on improving the quantum efficiency. The QE of rhombic and square structure is almost the same, which shows that the direction of the graphics does not affect QE, but the shape of the graphics has a greater impact. The QE peak value of ordinary device is about $30 \%$, that of square diffractive ring device is about $80 \%$, and that of circular diffractive ring device is about $60 \%$.

(a)

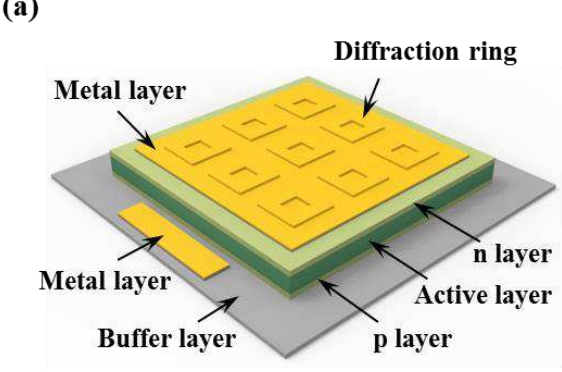

(c)

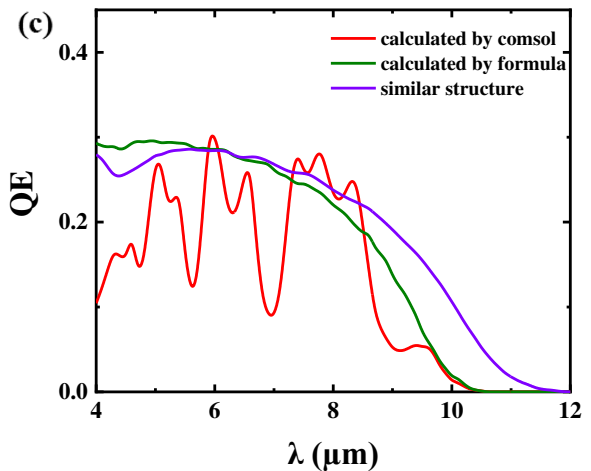

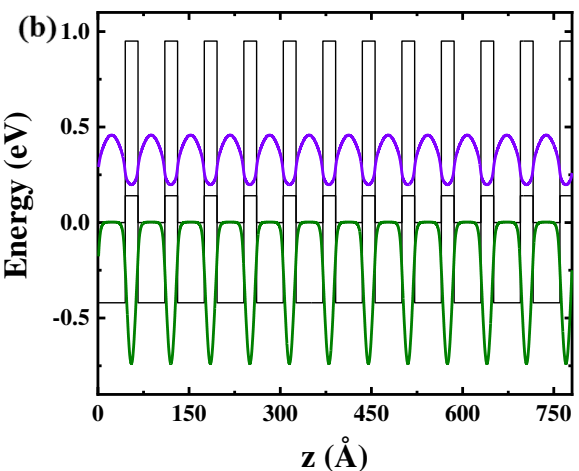

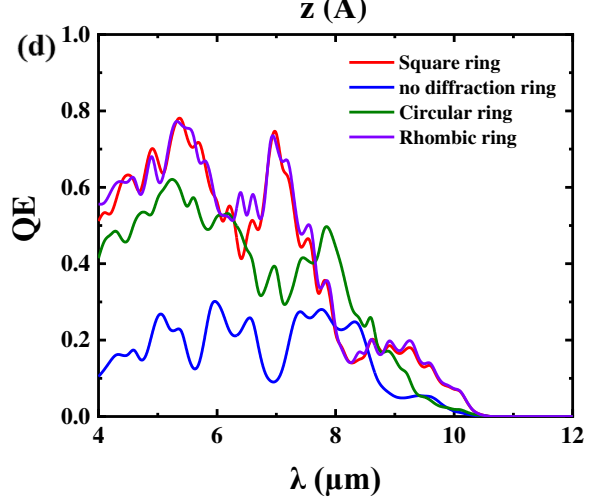

Fig. 1. (a) The structure diagram of the diffractive ring structure detector. (b) The wave function diagrams of the $\mathrm{C} 1$ and $\mathrm{HH} 1$ band of the absorption layer. (c) The QE curve of the device without diffraction ring simulated by COMSOL software compared with the results calculated by the formula and the materials with similar structure and thickness. (d) The comparison of QE between square, diamond and circular diffractive ring devices with the same diameter and non diffractive ring devices.

Fig. 2 and Fig. 3 show the electric field distribution of the device with different diffraction ring shapes at several incident light wavelengths. The cross sections of electric field distribution of square, circular, rhombic diffractive ring and non diffractive ring devices at the maximum value of electric field integration of $10.2 \mu \mathrm{m}$ are shown in Fig. 
2. At $10.2 \mu \mathrm{m}$, under the same legend, compared with the basic device, the electric field intensity of the device with diffraction ring increases at the interface between the absorption layer and the p-region, and the interface between the absorption layer and the n-region contact layer. And it is obvious that the electric field intensity of square and rhombic diffractive ring devices are larger. Fig. 3 (a) and (c) show the comparison of the electric field distribution between the square diffractive ring device and the non diffractive ring device at a QE peak of 7.2 $\mu \mathrm{m}$. In the ordinary device, the electric field intensity is low and evenly distributed. In the square diffractive ring device, the high electric field intensity is mainly at the edge of the diffractive ring and the metal layer, and the whole electric field of the device may be larger than that of the ordinary device due to the enhanced diffraction of light. Fig. 3 (b) and (d) show the comparison of the electric field distribution between the circular diffractive ring device and the non diffractive ring device at a QE peak of $8.1 \mu \mathrm{m}$. Similarly, in ordinary devices, the electric field intensity is low and the distribution is relatively uniform, and it is larger at the edge of the metal layer. In the circular diffractive ring device, the high electric field intensity is mainly concentrated in the diffractive ring, the inner part of the diffractive ring and the edge of the metal layer. The whole electric field of the device is larger than that of the ordinary device, but the distribution of the electric field intensity in the non diffractive ring is scattered, which is different from that of the square diffractive ring.

(a)

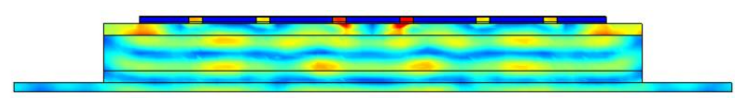

(c)

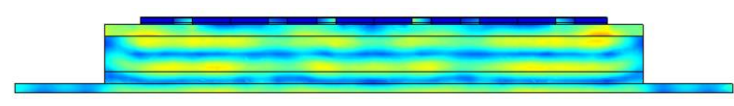

(b)

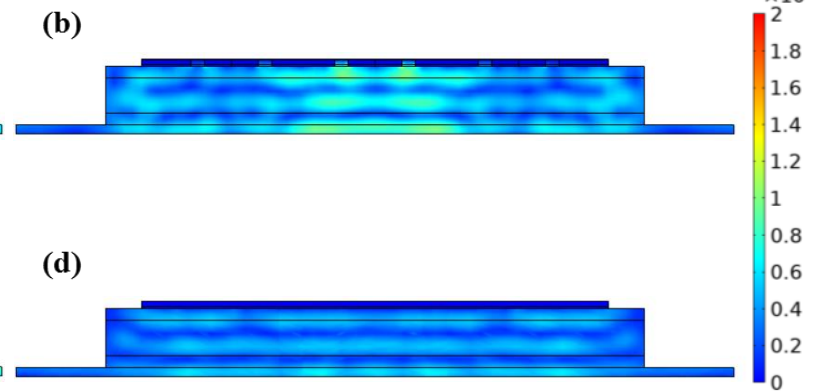

Fig. 2. The cross section of electric field distribution of (a) square diffraction ring, (b) circular diffraction ring, (c) diamond diffraction ring and (d) non diffraction ring at $10.2 \mu \mathrm{m}$.

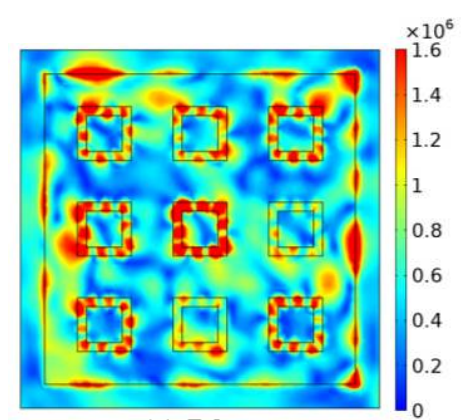

(a) $7.2 \mu \mathrm{m}$

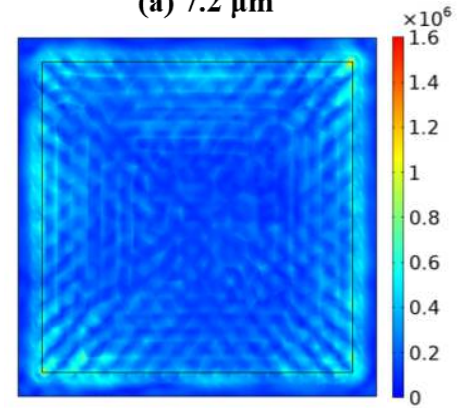

(c) $7.2 \mu \mathrm{m}$

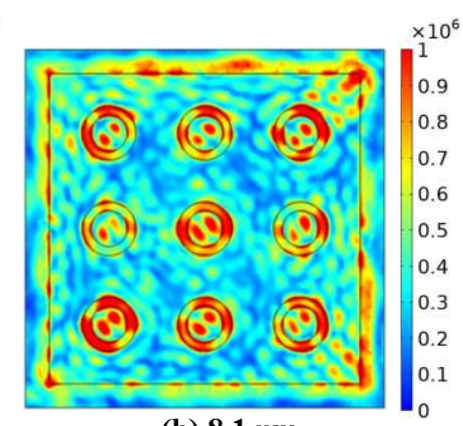

(b) $8.1 \mu \mathrm{m}$

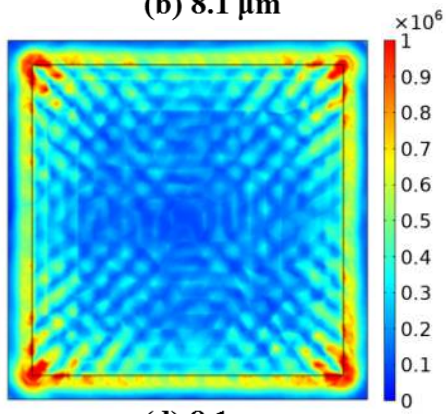

(d) $8.1 \mu \mathrm{m}$

Fig. 3. The top view cross section of electric field distribution of (a) square diffraction ring, (c) non diffraction ring device at $7.2 \mu \mathrm{m}$, and the top view cross section of electric field distribution of (b) circular diffraction ring and (d) 
non diffraction ring device at $8.1 \mu \mathrm{m}$.

From the above comparison, we can see that the square diffraction ring device has higher QE. Here we further analyze the influence of the size and position of the square diffraction ring. By changing the size of the diffraction ring, the effect on the QE of the device is studied, as shown in Fig. 4. Fig. 4 (a) shows that with the change of the outer diameter of the diffraction ring, the wavelength position corresponding to the peak value has a small shift, but the overall change is not big. At the maximum outer diameter of $7.5 \mu \mathrm{m}$, because the diffraction ring gradually becomes closer to the plane from the ring, QE gradually becomes flat, the peak value of the front wavelength becomes smaller, and the peak value of the back wavelength becomes larger. In fig. 4 (b), the difference between the inner and outer diameters of the diffractive ring is $1.5 \mu \mathrm{m}$. With the increase of the size of the diffraction ring, the positions of the peaks of QE hardly change, but the QE corresponding to the peaks and troughs change a little. In Fig. 4 (c), with the change of the spacing of nine diffraction rings with the size of $4.5 \mu \mathrm{m} / 3 \mu \mathrm{m}$, the positions of several main peaks in QE do not change much, but the wavelength positions corresponding to the small peaks beside QE change greatly. Figure 4 (d) shows the effect of the height change of the diffraction ring on QE. Because the diffraction ring is smaller than the thickness of the metal layer, the change range is very small, so the QE change amplitude in the figure is smaller than that in the first three figures. It can be seen from Figure 4 that the QE of the device will change according to the size of the diffraction ring, but the change range is small, which indicates that the square diffraction ring has good stability and high fault tolerance in the actual device preparation.
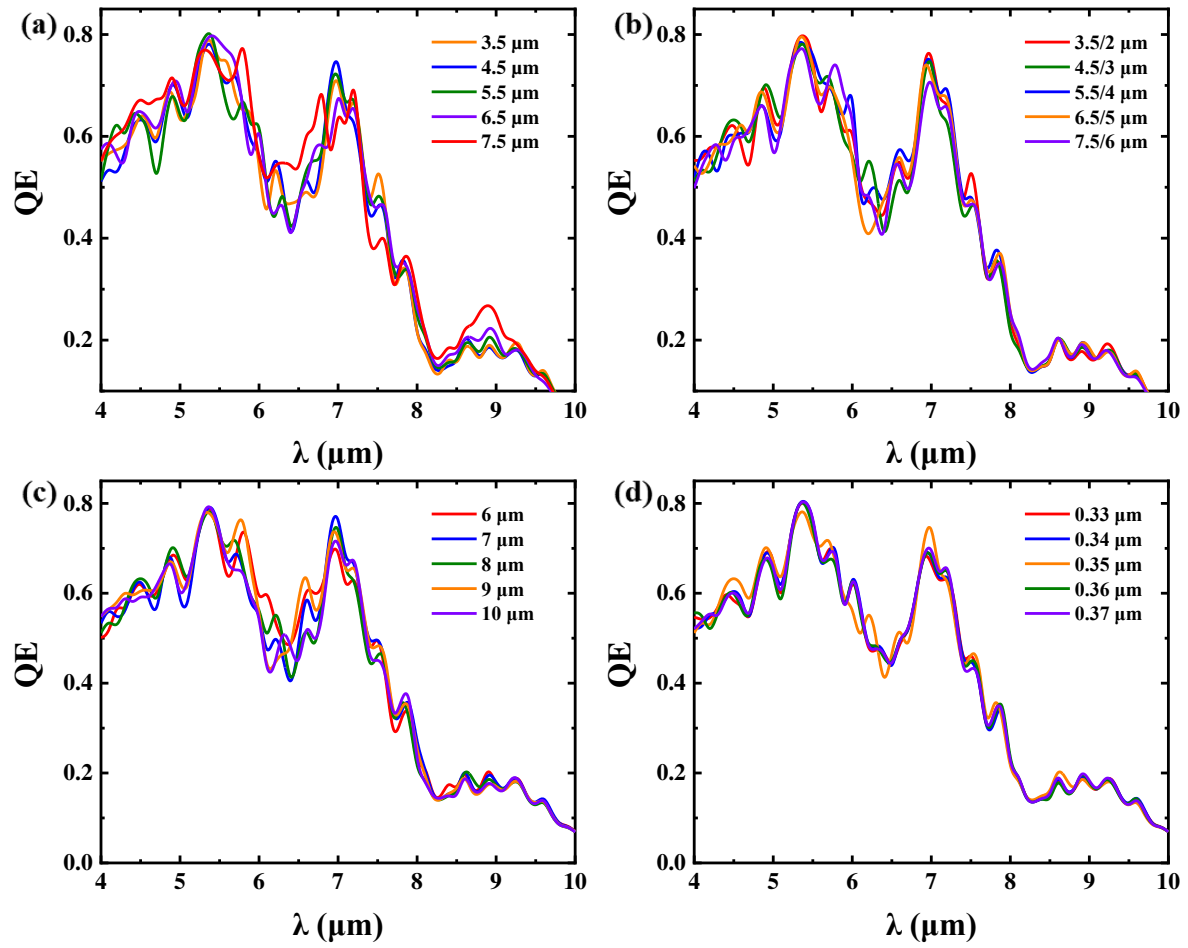

Fig. 4. The quantum efficiency curve of the detectors changes with (a) changing the size of the outer ring, (b) changing the size of the inner and outer diameter of the diffraction ring when the difference between the inner and outer diameters remains unchanged, (c) changing the spacing of nine diffraction rings, and (d) changing the height of the diffraction ring.

Furthermore, the influence of the thickness of the absorption layer on the QE of the device with square diffraction ring structure is calculated. Three absorption layer thicknesses of $975 \mathrm{~nm}$ (150 periods), $1950 \mathrm{~nm}$ (300 periods) and $2600 \mathrm{~nm}$ (400 periods) are selected for calculation. Fig. 5 shows the QE comparison of square, circular diffractive ring devices and non diffractive ring devices with three absorption layer thicknesses. In Fig. 5 (a), with the increase 
of the thickness of the absorption layer, the peak value of QE increases, and the QE near the cut-off wavelength increases as a whole, and the peak value decreases more steeply. However, at the incident light wavelength of $4 \mu \mathrm{m}$ $\sim 7 \mu \mathrm{m}$, the increase amplitude is not very large. According to the optical calculation of the Fabry Perot (F-P) cavity, when the cavity length changes from $L$ to $L+\Delta L$, the corresponding wavelength $\lambda$ becomes $\lambda+\Delta \lambda$. When the variation range of cavity length is less than the wavelength, the following relation is ${ }^{16}$,

$$
\frac{\Delta \lambda}{\lambda}=\frac{\Delta L}{L}
$$

Obviously, the length of the cavity raises with the increase of the thickness of the absorption layer, and the peak position of the oscillation wave increases accordingly. The device designed here includes the F-P cavity between each layer and the cavity formed between the surface air and the diffraction ring, so it is difficult to analyze the relationship between the specific position of the wave crest and the cavity length. It can be seen from Fig. 5 (a), (b) and (c) that with the increase of the thickness of the absorption layer, the QE near the cut-off wavelength increases as a whole, and the peak value decreases more steeply. In fig. 5 (b), QE of the circular diffractive ring device is smoother than that of the other two devices, and the difference between peak and valley is smaller.
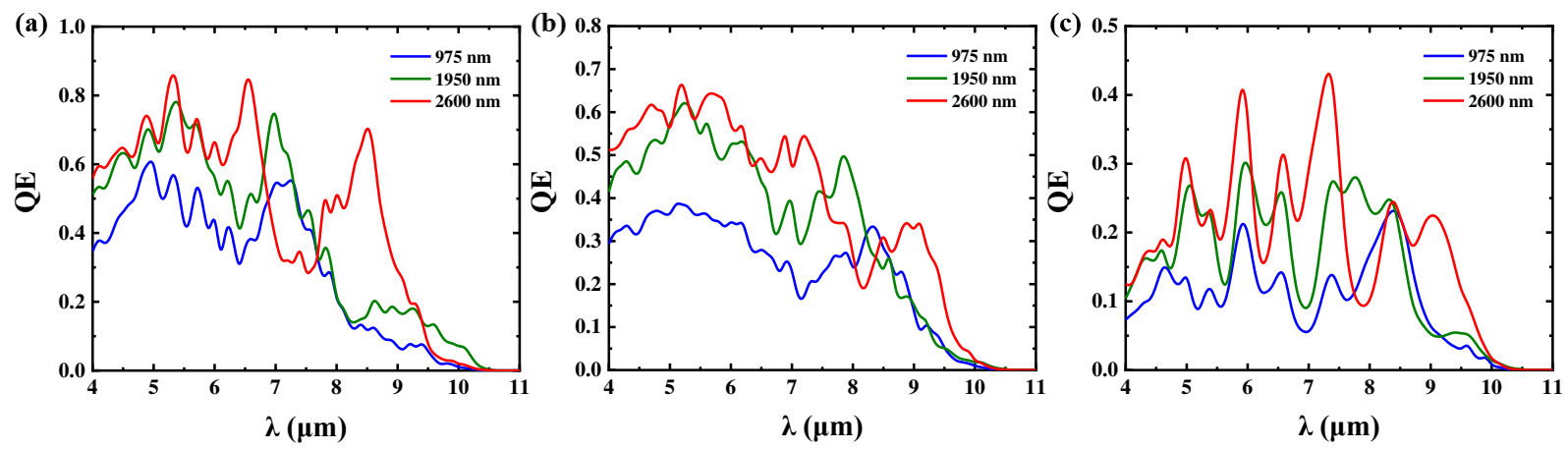

Fig. 5. QE curves of (a) square diffractive ring device, (b) circular diffractive ring device, and (c) non diffractive ring device with absorption layer thickness of $975 \mathrm{~nm}, 1950 \mathrm{~nm}, 2600 \mathrm{~nm}$, respectively.

In addition to $\mathrm{QE}$, dark current and specific detectivity are also important parameters to measure device performance. The dark current mechanism is analyzed here. Fig. 6 (a) shows the dark current mechanism of the detector with $1950 \mathrm{~nm}$ absorption layer thickness. At low negative bias, generation-combination dark current is dominant, followed by diffusion dark current, and band to band tunneling dark current and trap assisted tunneling dark current are dominant at higher voltage. Photovoltaic devices mainly work at low negative bias voltage, so generation-combination dark current and diffusion dark current are the main optimization objectives of devices. Fig. 6 (b) shows the total dark current curve of the device with three kinds of absorption layer thickness. With the increase of absorption layer thickness, the dark current gradually increases.
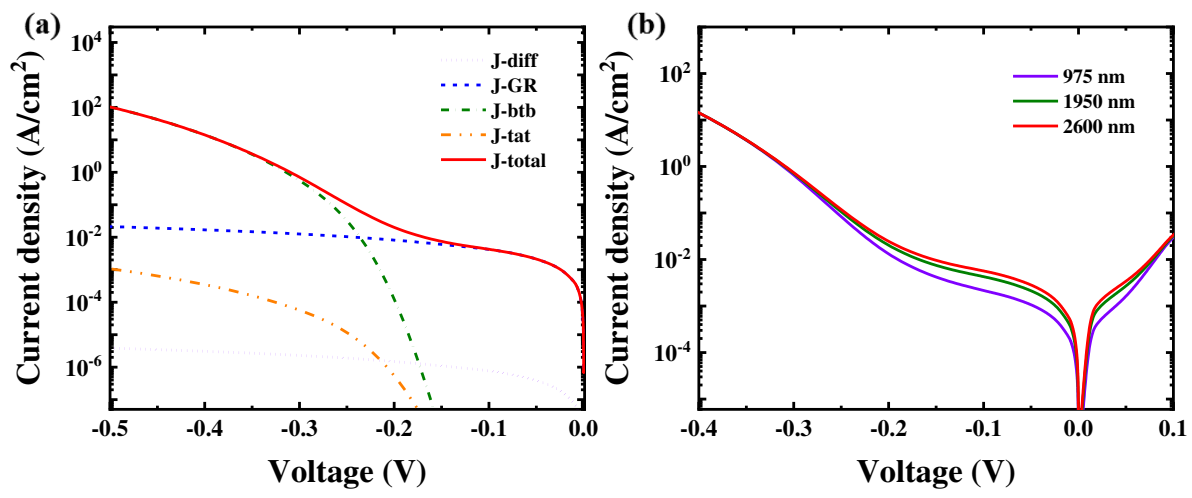
Fig. 6. (a) Each dark current and total dark current curve of the device with $1950 \mathrm{~nm}$ absorption layer. (b) The total dark current curves of the devices with the thickness of $975 \mathrm{~nm}, 1950 \mathrm{~nm}$ and $2600 \mathrm{~nm}$.

The differential resistance area product under zero bias $\left(\mathrm{R}_{0} \mathrm{~A}\right)$ is calculated by $\mathrm{I}-\mathrm{V}$ relation, and the specific detectivity of the device is calculated by formula (11). The specific detectivity of square, circular and non diffractive ring devices with three absorption layer thicknesses of $975 \mathrm{~nm}, 1950 \mathrm{~nm}$ and $2600 \mathrm{~nm}$ is shown in Fig. 7. It can be seen that the change of specific detectivity is basically consistent with QE, but the curve is smoother. The specific detectivity of the device with diffraction ring is about one order of magnitude higher than that of the device without diffraction ring.
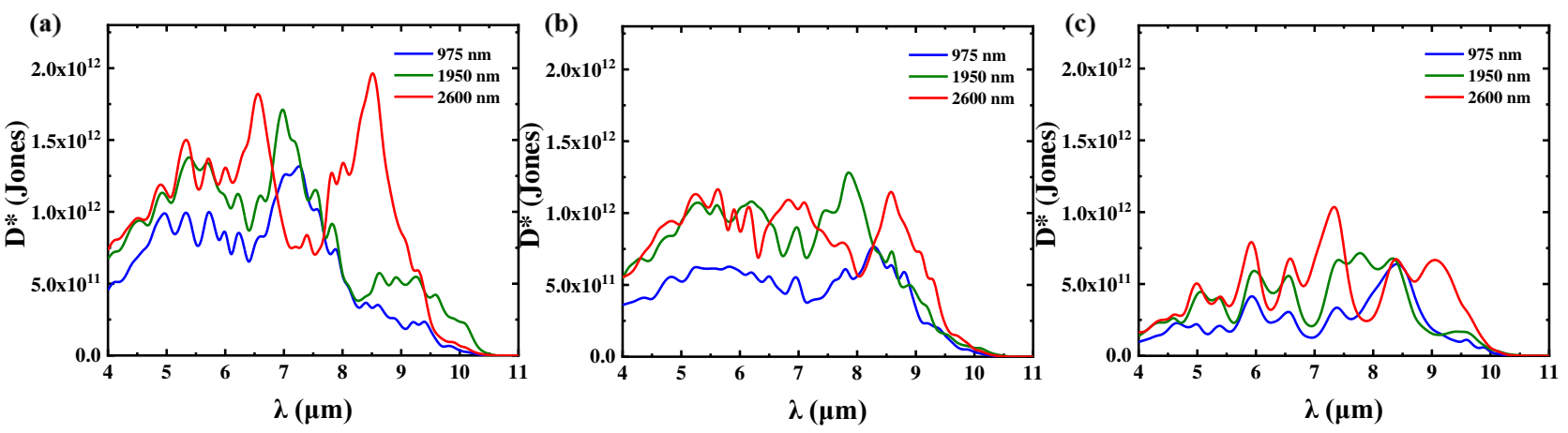

Fig. 7. The specific detectivity curves of (a) square diffractive ring device, (b) circular diffractive ring device, and (c) non diffractive ring device with absorption layer thickness of $975 \mathrm{~nm}, 1950 \mathrm{~nm}, 2600 \mathrm{~nm}$, respectively.

\section{Conclusions}

In this paper, a systematic theoretical model is established to calculate the QE of the device with diffraction ring and the ordinary device. The influence of the shape and size of the diffraction ring and the thickness of the absorption layer on the device parameters is analyzed. By using the electromagnetic field model, the peak QE of the ordinary device is about $30 \%$, the peak $\mathrm{QE}$ of the device with square diffraction ring is about $80 \%$, and the peak $\mathrm{QE}$ of the device with circular diffraction ring is about $60 \%$. It shows that the $\mathrm{QE}$ of the device can be significantly improved by adding diffraction ring. And it is obvious that the optimization effect of the square diffraction ring is better than that of the circular diffraction ring, and it is more insensitive to the size change. Therefore, the square diffraction ring structure is suitable for the fabrication of practical devices. At the same time, the dark current and specific detectivity of the device with the change of the thickness of the absorption layer are analyzed. In PIN photodetectors, the dominant dark current mechanisms include generation-recombination dark current and diffusion dark current at low negative bias voltage. The specific detectivity of the device with diffraction ring is about one order of magnitude higher than that of the ordinary structure without diffraction ring.

\section{Acknowledgements}

This work was supported by National Natural Science Foundation of China (Grant No. 61835011). National Natural Science Foundation of China (Grant No. U1431231). National Natural Science Foundation of China (Grant No. 12075244). Key Research Projects of the Frontier Science of the Chinese Academy of sciences (No. QYZDY-SSWJSC004). Beijing Science and Technology Projects (Grant No. Z151100001615042). National Key Research and Development Plan (No. 2016YFB0402402 and No. 2016YFB0400601). National Basic Research Program of China (973 Program) (No. 2015CB351902). National Science and Technology Major Project (2018ZX01005101-010).

\section{Author contributions statement}


Yanan Du conceived and designed of the work, established a theoretical model, captured, analyzed, and interpreted the data, wrote the initial draft ; Xu Yun commented and revised the first draft, and provided computational resources and analytical tools; Bo Cheng established a theoretical model; Guofeng Song provides computational resources and analytical tools. All authors reviewed the manuscript.

\section{Competing interests}

The author(s) declare no competing interests.

\section{Availability of materials and data}

All data generated or analysed during this study are included in this published article.

\section{References}

1 Sai - Halasz, G. A., Tsu, R. \& Esaki, L. A new semiconductor superlattice. Appl. Phys. Lett. 30, 651-653, doi:10.1063/1.89273 (1977).

2 Salihoglu, O. et al. Electronic and optical properties of $4.2 \mu \mathrm{m}$ "N" structured superlattice MWIR photodetectors. Infrared Phys. Technol. 59, 36-40, doi:10.1016/j.infrared.2012.12.007 (2013).

3 Rogalski, A., Martyniuk, P. \& Kopytko, M. InAs/GaSb type-II superlattice infrared detectors: Future prospect. Applied Physics Reviews 4, doi:10.1063/1.4999077 (2017).

4 Sun, J., Choi, K. K., Olver, K. A. \& Fu, R. X. in Infrared Sensors, Devices, and Applications Viii Vol. 10766 Proceedings of SPIE (2018).

5 Safa, S. \& Asgari, A. Effects of ionized impurity and interface roughness scatterings on the electron mobility in InAs/GaSb type II superlattices at low temperatures. Modern Physics Letters B 30, doi:10.1142/s021798491650384x (2016).

6 Qiao, P.-F., Mou, S. \& Chuang, S. L. Electronic band structures and optical properties of type-II superlattice photodetectors with interfacial effect. Opt. Express 20, 2319-2334, doi:10.1364/oe.20.002319 (2012). Yang, Q. K., Fuchs, F., Schmitz, J. \& Pletschen, W. Investigation of trap-assisted tunneling current in InAs/(Galn)Sb superlattice long-wavelength photodiodes. Appl. Phys. Lett. 81, 4757-4759, doi:10.1063/1.1529306 (2002). Mohseni, H. et al. in Photodetectors: Materials and Devices V Vol. 3948 Proceedings of the Society of PhotoOptical Instrumentation Engineers (Spie) 153-160 (2000).

$9 \quad$ Nguyen, J. et al. Dark current analysis of InAs/GaSb superlattices at low temperatures. Infrared Phys. Technol. 52, 317-321, doi:10.1016/j.infrared.2009.05.022 (2009).

10 Delmas, M., Rossignol, R., Rodriguez, J. B. \& Christol, P. Design of InAs/GaSb superlattice infrared barrier detectors. Superlattices Microstruct. 104, 402-414, doi:10.1016/j.spmi.2017.03.001 (2017).

$11 \mathrm{Wu}, \mathrm{H}$. et al. Performance analysis of an N-structure type-Il superlattice photodetector for long wavelength infrared applications. Journal of Alloys and Compounds 684, 663-668, doi:10.1016/j.jallcom.2016.05.229 (2016).

12 Taghipour, Z., Kazemi, A. \& Myers, S. in Infrared Sensors, Devices, and Applications Vii Vol. 10404 Proceedings of SPIE (2017).

$13 \mathrm{Li}$, L. et al. Studying the effect of material parameters on detectivity in a p-n In0.53Ga0.47As photovoltaic detector. Solid-State Electronics 52, 11-16, doi:10.1016/j.sse.2007.07.032 (2008).

14 Choi, K. K. et al. Resonant structures for infrared detection. Applied Optics 56, B26-B36, doi:10.1364/ao.56.000b26 (2017).

15 Mou, S., Li, J. V. \& Chuang, S. L. Quantum Efficiency Analysis of InAs-GaSb Type-II Superlattice Photodiodes. IEEE J. Quantum Electron. 45, 737-743, doi:10.1109/jqe.2009.2013149 (2009).

16 Zhang, Y. et al. High-temperature fiber-optic Fabry-Perot interferometric pressure sensor fabricated by femtosecond laser. Opt. Lett. 38, 4609-4612, doi:10.1364/ol.38.004609 (2013). 


\section{Figures}

(a)
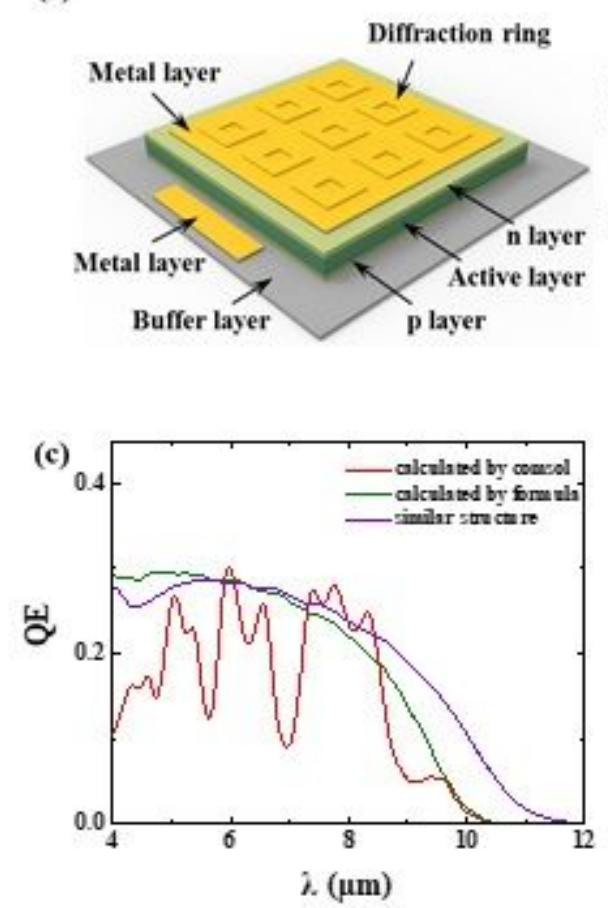
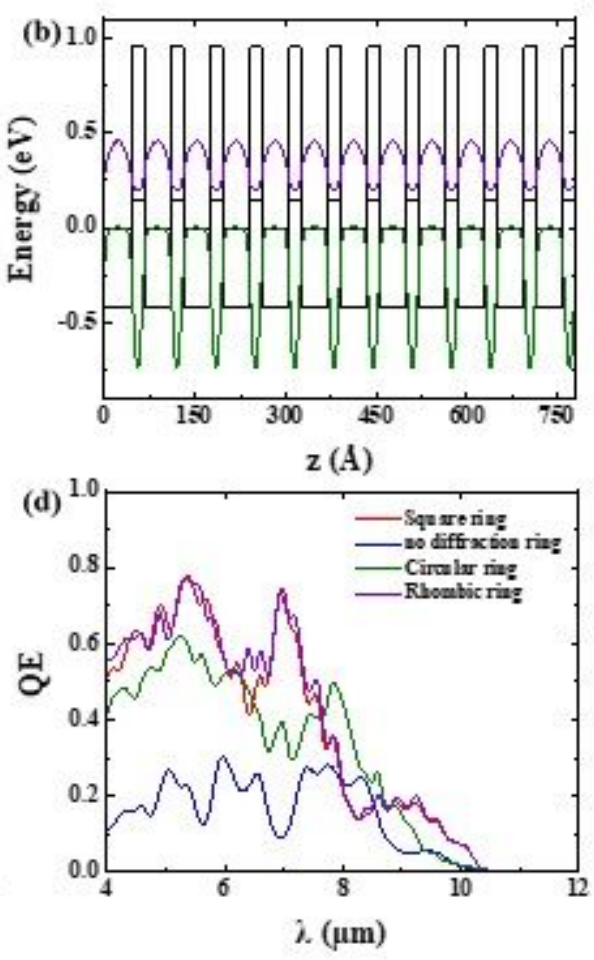

\section{Figure 1}

a) The structure diagram of the diffractive ring structure detector. (b) The wave function diagrams of the $\mathrm{C} 1$ and $\mathrm{HH} 1$ band of the absorption layer. (c) The QE curve of the device without diffraction ring simulated by COMSOL software compared with the results calculated by the formula and the materials with similar structure and thickness. (d) The comparison of QE between square, diamond and circular diffractive ring devices with the same diameter and non diffractive ring devices.

(a)

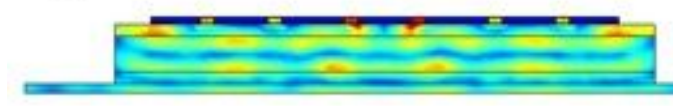

(c)

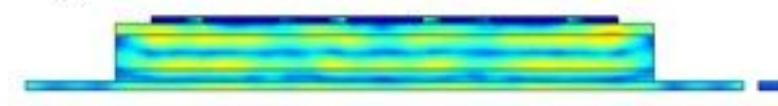

(b)
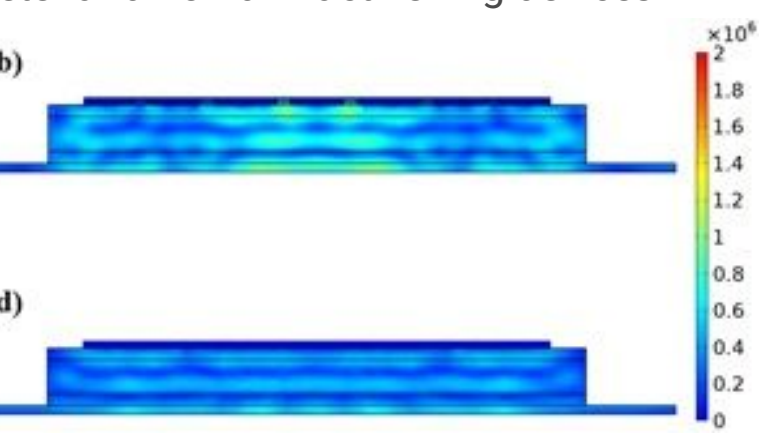

\section{Figure 2}

The cross section of electric field distribution of (a) square diffraction ring, (b) circular diffraction ring, (c) diamond diffraction ring and (d) non diffraction ring at $10.2 \mu \mathrm{m}$. 


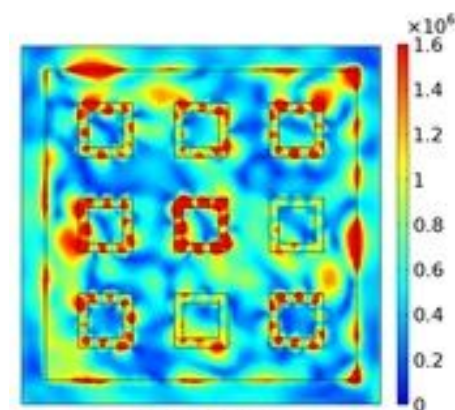

(a) $7.2 \mu \mathrm{m}$

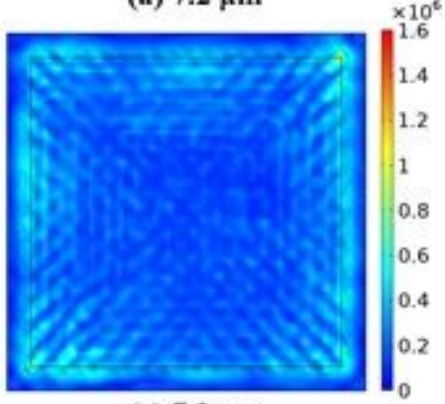

(c) $7.2 \mu \mathrm{m}$

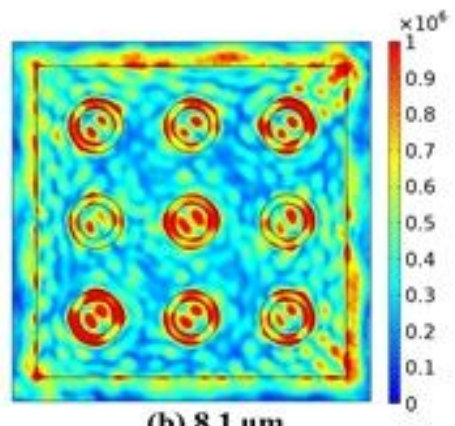

(b) $8.1 \mu \mathrm{m}$

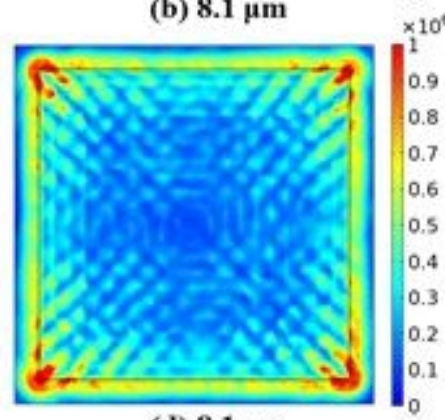

(d) $8.1 \mu \mathrm{m}$

\section{Figure 3}

The top view cross section of electric field distribution of (a) square diffraction ring, (c) non diffraction ring device at $7.2 \mu \mathrm{m}$, and the top view cross section of electric field distribution of (b) circular diffraction ring and (d) non diffraction ring device at $8.1 \mu \mathrm{m}$.
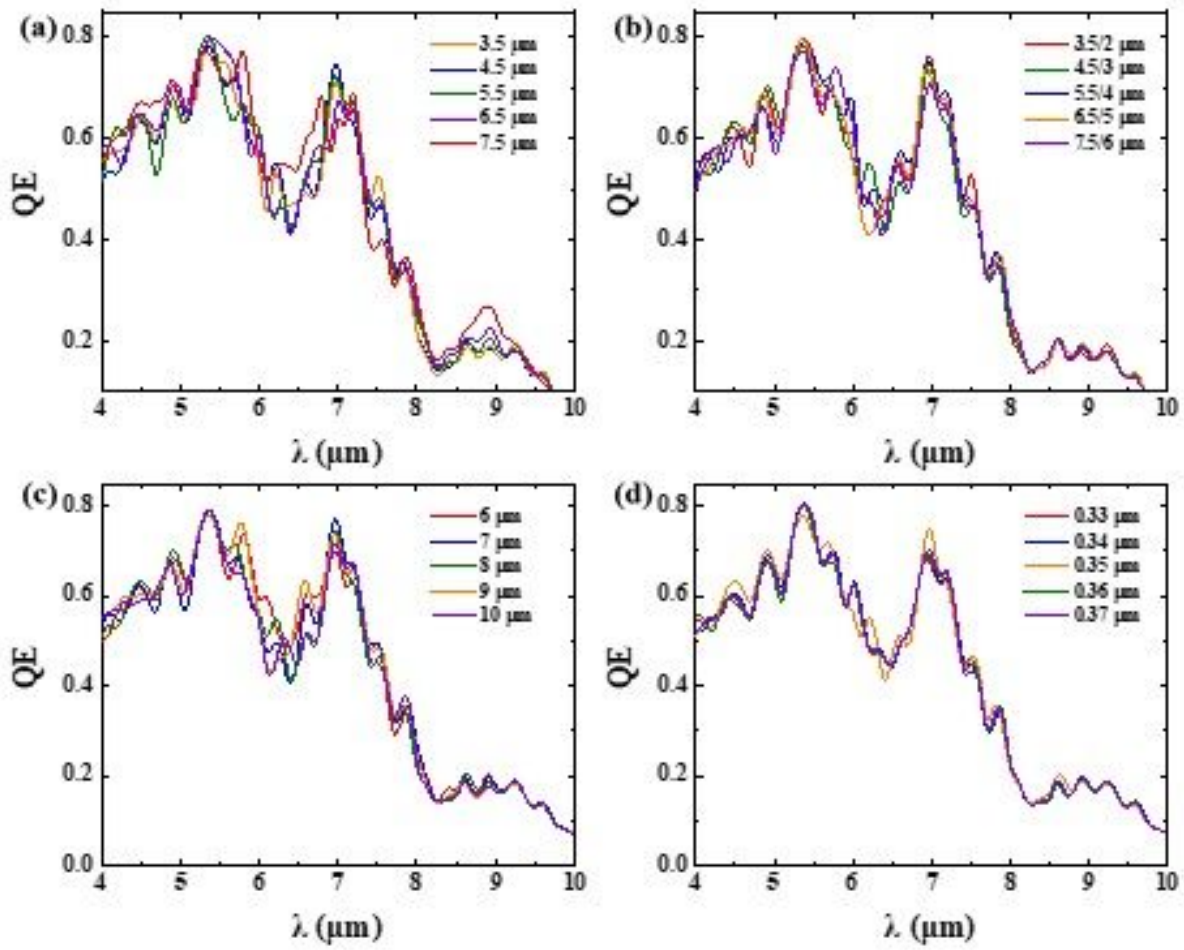

Figure 4 
The quantum efficiency curve of the detectors changes with (a) changing the size of the outer ring, (b) changing the size of the inner and outer diameter of the diffraction ring when the difference between the inner and outer diameters remains unchanged, (c) changing the spacing of nine diffraction rings, and (d) changing the height of the diffraction ring.
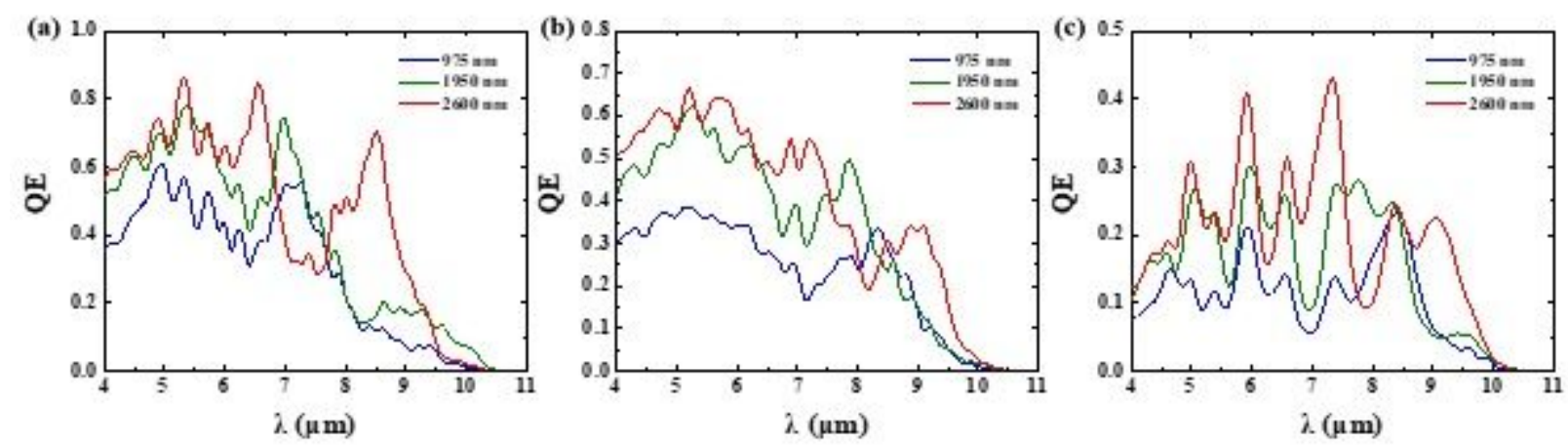

\section{Figure 5}

QE curves of (a) square diffractive ring device, (b) circular diffractive ring device, and (c) non diffractive ring device with absorption layer thickness of 975 nm, 1950 nm, 2600 nm, respectively.
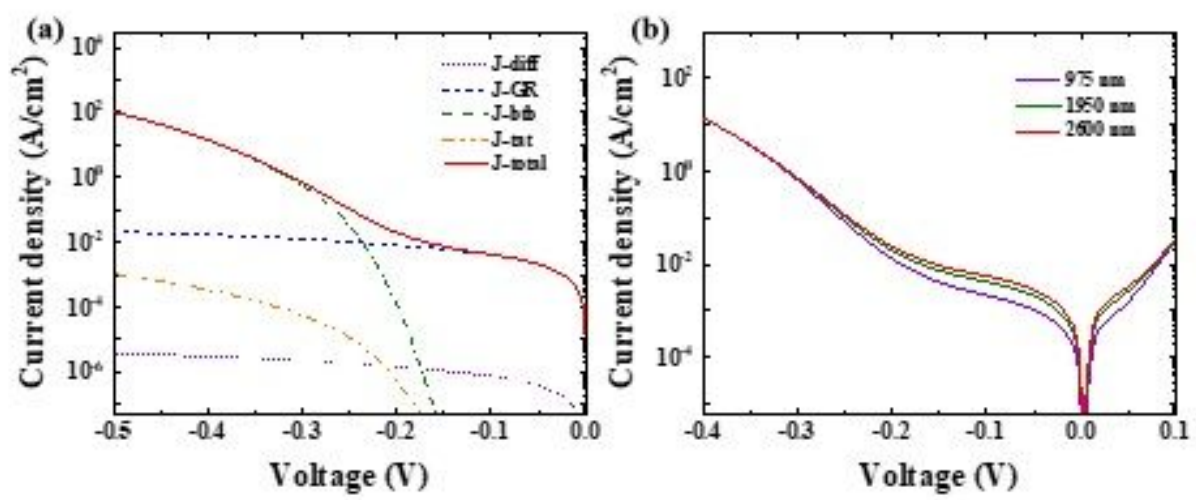

Figure 6

(a) Each dark current and total dark current curve of the device with $1950 \mathrm{~nm}$ absorption layer. (b) The total dark current curves of the devices with the thickness of $975 \mathrm{~nm}, 1950 \mathrm{~nm}$ and $2600 \mathrm{~nm}$.
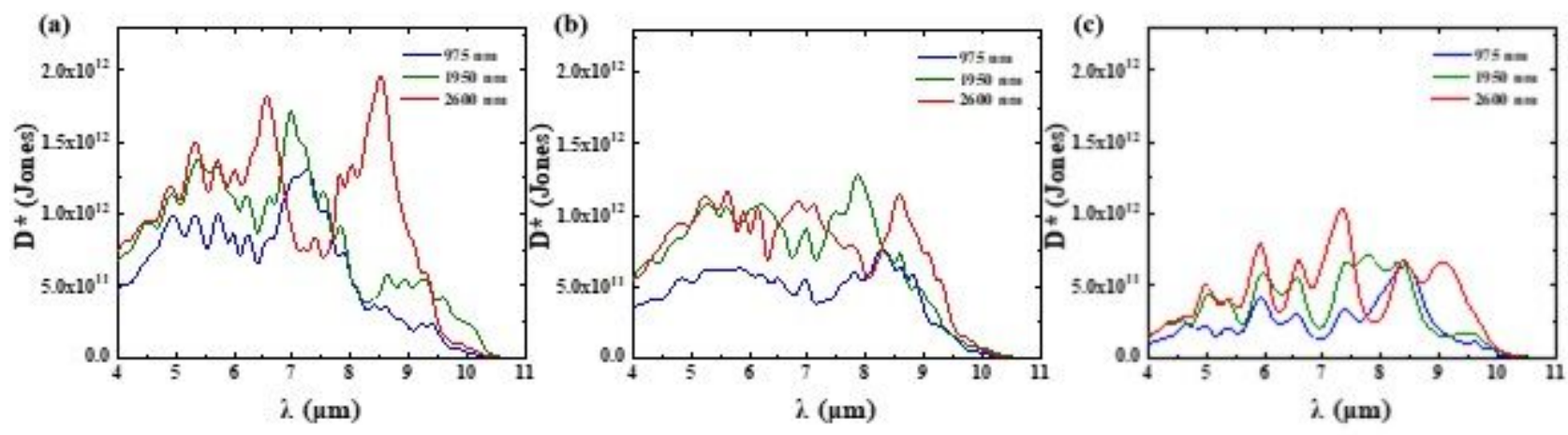

Figure 7 
The specific detectivity curves of (a) square diffractive ring device, (b) circular diffractive ring device, and (c) non diffractive ring device with absorption layer thickness of $975 \mathrm{~nm}, 1950 \mathrm{~nm}, 2600 \mathrm{~nm}$, respectively. 